\title{
Anaesthesia and genetics: still, the uncharted territories!!
}

Volume 4 Issue I - 2016

Keywords: Anesthesia; Genetics; Relevance; Impact on; Future considerations

\section{Editorial}

"All humans are born equal!" is an often quoted and sociologically pertinent axiom, irrespective of race, color, creed, caste, religion and many variations. It is absolutely true even from scientific point of view, as all of us, human beings are supposed to share $99.9 \%$ of our genetic material. So genotypically, we are all, almost similar. It is only $0.1 \%$ variabilityin the terms of diversity or 'polymorphisms' either in the non-coding or coding regions of DNA which differentiates us from one another. Some of these variants exhibit concealedor overt signs/ symptomsleading to expression of various genetically transmitted diseases phenotypically. ${ }^{1}$

These variants in the human genome result in variations in terms of various biologically active molecules, which play a major role in how an individual allows the drugs to behave, both in terms of pharmacokinetics and pharmacodynamics. ${ }^{2}$ So by corollary these variations contribute to an individual's sensitivity and response to a variety of drugs utilized for anesthetic practice. Variations may occur involving insertions or deletions at single or multiple nucleotides. As a result any variation in the expression or function of various enzymes/polypeptides, neuromodulators/transmitters and other active moleculesplaysa major role in the anaesthetic practice, which is the most crucial and acute application of clinical pharmacotherapeutics.

Logically, it becomes absolutely imperative on the part of practicing anesthesiologist to be well acquainted with common and not so common genetically transmitted conditions, heredito-familial disorders, chromosomally propagatedtraits and even unexpressed (recessive) characteristics because any of these may have an influence on the anesthetic/perioperative pharmacological management. They may, at one end of the spectrum simply, mildly influence the effect of these drugs on the patient and on the other end, result in extremely and potentially dangerous, life threatening consequences. With this background in mind let us embark upon understanding the finer nuances of this very exciting and rapidly evolving field of pharmacogenetics/genomics and its relevance in our day to day anesthesia practice.

Early insights into the clinical impact of pharmacogenetics were provided by anesthesiology. The in-depth investigations into prolonged apnea after succinylcholine administration, thiopentoneinduced porphyria and malignant hyperthermia contributed to the novel science of pharmacogenetics in the early 1960s. Genetic polymorphisms involved in pharmacokinetics (absorption, distribution, metabolism, and excretion of drugs i.e. what the human body does to the drugs) and pharmacodynamics (receptors, ion channels and enzymes, i.e. what drugs do to the human body) can affect an individual's response to the anesthetic agents. In addition, genetic variation in proteins/polypeptides, directly unrelated to drug action or metabolism, can influence responses of the individual to the

\author{
Mridul M Panditrao, Minnu M Panditrao \\ Professor, Head and In-charge of ICU, Department of \\ Anaesthesiology and Intensive Care, Adesh Institute of Medical \\ Sciences and Research (AIMSR),Adesh University, India
}

Correspondence: Mridul M Panditrao, Professor, Head and In-charge of ICU, Department of Anaesthesiology and Intensive Care,Adesh Institute of Medical Sciences and Research (AIMSR), Dean Academic Affairs, Adesh University, Bathinda, Punjab, India, Tel +91 8699921884, Email drmmpraol@gmail.com

Received: December 26, 2015 | Published: January 04, 2016

environmental changes that occur during anesthesia. This review will summarize the current knowledge of genetic variations in responses to drugs relevant to anesthesia, and how this impacts its clinical practice.

Thus when considering the factors influencing these variations, along with age, sex, body weight etc., the genetic profile also must be given important consideration as this may have major influence on the pharmacological needs of the individual . ${ }^{3}$

\section{Genetic disorders}

As it was mentioned above, genetic disorders result from abnormalities within genes or chromosomes. Mutation inside the genes, deletion of specific genes, chromosomal aberrations or other mechanisms such as two recessive genes coming together or exposure to radiation can all lead to genetic disorders. Inherited genetic disorders can be identified either immediately after the birth or later on in life. Presently, there are over 4,000 diagnosed genetic disorders, although new disorders are constantly being discovered.

Types of genetic disorders: Genetic disorders include five types: single gene disorders, polygenic disorders, multifactorial problems, chromosomal abnormalities and mitochondrial mutations.

Single gene disorders: Single gene disorders, also called Mendelian or monogenic disorder, are caused by changes or mutations within the DNA sequence of a single gene. Single gene disorders have higher risk of inheritance. Single gene disorders include:

i. Autosomal disorders-such as achondroplasia which is defective bone development that causes dwarfism. Autosomal disorders are caused by abnormal gene of one parent.

ii. Autosomal recessive disorders-such as sickle cell anemia and cystic fibrosis, which occur due to abnormal genes of both parents.

iii. X-linked disorders-include disorders such as hemophilia caused by single abnormal gene on the $\mathrm{X}$ chromosome.

iv. Y-linked disorders-mutation or abnormality is present only in $\mathrm{Y}$ chromosome. These disorders are passed from father to son. 
Polygenic disorders: A genetic disease caused by the combined actions of two or more genes. Examples of polygenic diseases include coronary heart disease, diabetes, hypertension and peptic ulcers. Because such disorders depend on the simultaneous presence of several genes, they are not inherited in simple Mendelian patterns.

Multi factorial problems: These problems result from combination of environmental factors and mutations in multiple genes. These disorders include heart disease, Hypertension, diabetes, obesity, asthma, autism, arthritis, cancer, ciliopathies, cleft palate, mental retardation.

Chromosomal abnormalities: These disorders occur due to abnormalities in number of chromosomes or structure of chromosomes and they are generally caused by problem with cell division. Chromosomal abnormalities include disorders such as Down's syndrome, Turner's syndrome, chromosomal sequence deletions, inversions and translocations.

Mitochondrial mutations: Mitochondrial mutations are always inherited from the female parent because mitochondria are inside the egg cells during fertilization. Mitochondrial mutations include Leber's hereditary optic neuropathy, MERRF (type of epilepsy), Leigh's syndrome and NARP.

One can broadly differentiate the correlation between genetics and anesthesia, under two broad headings:

i. How does the genetics or any change in the basic genetic makeup in a human being affect thePre-operative assessment/ preparation, Anaesthetic/ intra operative management and Post-operative care, In other words, the entire spectrum of Anesthetic care?

ii. How do the anaesthetic agents and anaesthesia management influence genetic makeup of the humans exposed to it, e.g. what are the various genetic disorders, which may be the result of exposure to anaesthetic/ sedative drugs, especially during the intra-uterine life?

I. Influence of Genetic Expression/ Genetic disorders on Anaesthetic management: The changes which take place in the genetic makeup of any individual and when expressed as a developmental anomaly, are bound to affect the anaesthetic management. ${ }^{4}$ These patients with common and not so common genetic disorders, with single or multiple congenital abnormalities are very challenging for anaesthetic management. These abnormalities can be in the form of anatomic and physiologic changes that take place as a result of either single gene or multiple gene mutations. As a result it is not only from the pharmacological or biochemical, even from the structural, functional and procedural point of view, that these may pose challenges. So an attempt is being made to highlight the specific problem/s, the anaesthesiologist will be facing and how to tackle that, rather than concentrating on specific syndromes or the complexities of heredito-familial and genetic expression of the disease.

II. The important concerns, one has to consider are as follows ${ }^{5}$

A. Airway Abnormalities/ difficulties: The major concern for any anaesthesiologist is the anticipated airway difficulty, due to anatomical/ developmental defects and anomalous airway related structural (cranio-facial) problems. These defects can be as follows: In patients with Cleft lip/cleft palate, various trisomy micrognathia (hypoplastic mandible) and relative or obvious macroglossa (glossoptosis) are some of the commoner defects. Not so common or rarer defects may be choanal atresia, trachea- oesophageal fistula. Some of the examples of these defects are as follows: Achondrogenesis types I, II, Apert syndrome, Alagille syndrome, Baller Gerold syndrome, Cat's Eye syndrome, Coffin Siris syndrome, Escobar syndrome, Aminopterine methotrexate syndrome, Foetal Hydantoin syndrome/ dilantin syndrome, Fryn's syndrome, foetal trimethadione syndrome, fronto-nasal dysplasis syndrome Baller Gerold syndrome, Cat Eye, Goldenhar syndrome, Gorlin syndrome, haywell's syndrome, Jarcho-Levin syndrome, Klippel- Feil syndrome, Mardon-Walker, Marfan's, Miller, Meckel-gruber, Mohar's syndrome, Nager's syndrome, Noonan's syndrome,Peters-plus syndrome, Pierre Robin, Proteus syndrome, Seckell syndrome, stickler syndrome, Trecher-collin's syndrome, Various trisomies, 8mosaic, 9mosaic, 13, 18, Wander woude syndrome. XXXY and XXXXY syndrome.

B. Congenital/developmental cardiac defects: This is the second most common set of anomalies, usually found in association with other sets of developmental abnormalities especially, craniofacial, vertebra-skeletal, rarely thoracic. The examples of some these defects are as follows: Antley-Bixler syndrome, Aperts, Angelman syndrome, Alagille syndrome, Cat's Eye, Cohen, Carpenter, Costello's syndrome, Foetal Hydantoin syndrome/ dilantin syndrome, Fryn's syndrome, foetal trimethadione syndrome, Goldenhar syndrome Haywell's syndrome, Miller syndrome, Klippel-Feil syndrome, Phenyl-ketonuria, Stickler syndrome, trisomies 8 and 9, Turner's WAGR and Williams syndrome.

C. Renal/ hepatic: These are found very rarely singly, but always in association with many other systemic problems. These invariably complicate the peri-operative management. The examples of some these defects are as follows: Acrodystosis, Alpha Thallasemia, Angelman syndrome, Antley- Bixler, Apert syndrome, Baller- Gerold, Cat's Eye syndrome, Branchio- occulo facial syndrome, Foetal Hydantoin syndrome/ dilantin syndrome, Fryn's syndrome, foetal trimethadione syndrome, Goldenhar syndrome, Klippel Feil syndrome, Miller syndrome, Turner syndrome and WAGR syndrome.

D. CNS/ Mental retardation: Mental retardation is a classical feature of many chromosomal anomalies as well as in association with various other systemic problems. The examples of some these defects are as follows: Down's syndrome, Acrodystosis, Alpha, Thalassemia, Angelman's syndrome, Cowden syndrome, Costello syndrome, Watson syndrome.

\section{Anaesthestic considerations}

The anaesthetic problems are co-existence of Micrognathia, Coarctation of aorta, Horse shoe kidney, Thoracic cage and vertebral anomalies, especially in Turner's syndrome. In most of the patients airway is a major problem. In Goldenhar's syndrome thorough evaluation of upper and lower airway, clinically as well as radiologically is required. Systemic function tests viz; pulmonary, hepatic and renal, avoiding use of Neuro muscular blocking drugs is important. Anticholinergic drugs, if required to be used in very small quantity and sparingly. Intra venous induction with ketamine or thiopentone is much more desirable than inhalational one. Post-operative Critical care is absolutely essential. The patients with Klippel- Feil present unique problem due to extremely short neck and fusion of cervical vertebrae, associated with other vertebral and cardiac anomalies. It is necessary to have thorough airway assessment before anaesthetic plan. In addition vertebral defects make Neuraxial block very difficult. In patients with Noonan syndrome, GA may be best avoided, because 
of possibility of precipitating Malignant Hyperpyrexia (MH), where regional techniques may be the only suitable/available alternatives. On the other hand, in Sickle cell disease patients prevention of hypoxia, hypercarbia, dehydration, hypothermia and acidosis is essential to avoid the sickling and development of crisis during the surgical procedure.General anaesthesia would be the procedure of choice.

While in Neurofibromatosis (NF) complex, inhalational induction with sevoflurane is recommended ${ }^{6}$ there is a lot of evidence suggesting enhanced sensitivity of NFI patients to non-depolarizing muscle relaxant drugs..$^{7-9}$ Other associated abnormalities may also contribute to the difficulties. Pulmonary fibrosis increases the risk, with its complications of gaseous exchange. Larger $\mathrm{FiO} 2$ may have to provided intra as well as post operatively. So it is obvious to us that, irrespective of type of mutation, transmission, presentation or association of various genetic and clinical features, these disorders pose a major challenge to anaesthesiologist.

III. How do the anaesthetic agents and anaesthesia exposures influence the genetic makeupof an individual especially in Intra Uterine life/ developing fetus?: This has always been a very controversial, delicate yet important aspect. There is no clear opinion or evidence regarding any of the anaesthetic agents being directly involved in development of mutagenicity or teratogenicity in human beings exposed to the anaesthetic agents. There are reports of Teratogenic changes being observed in rats exposed to nitrous oxide for prolonged periods. The critical period was considered to be 8th Intra uterine day in rats, which was considered to correspond to that of 6 th week in human embryo. ${ }^{10}$ However there is no evidence of similar effects being seen in human beings. It had been suggested in past to avoid exposure to nitrous oxide in early pregnancy, which has become debatable now. ${ }^{11}$

However recently published studies by Kallen and Mazze ${ }^{12}$ and by Sylvester et al. ${ }^{13}$ suggest that the newborns of females exposed to general anaesthesia in first trimester had more incidence of neural tube defects, hydrocephalous and eye anomalies especially cataracts. On the other hand there are multiple reports which put forward of having no teratogenicity.

Such information regarding other anaesthetic has not been found inspite of detailed literature search. So one is inclined to think aloud that, the fear of the teratogenicity and mutagenicity in pregnant females, especially pregnant Operating Room (OR) personnel has reached a stage of paranoia. One must use a prudent judgment in weighing pros and cons of exposing these patients to drugs like nitrous oxide in early pregnancy.

\section{Future considerations: pharmacogenomics}

Definition: Pharmacogenomics is the study of how an individual's genetic inheritance affects the body's response to drugs. It is a portmanteau of pharmacology and genomics and is the study of the role of genetics in drug response. It deals with the influence of acquired and inherited genetic variation on drug response in patients by correlating gene expression or single-nucleotide polymorphisms with drug absorption, distribution, metabolism and elimination, as well as drug receptor target effectsi.e. pharmacokinetics and pharmacodynamics. ${ }^{14}$ As it is the intersection of pharmaceuticals and genetics and as we all know anaesthesia is nothing but clinical pharmacology completely embodied and crystallized into the real time application to achieve very specific precise and result oriented goals, Pharmacogenomics can assume a great value in the context of Anesthesia, as principles and practices of this science are also the same.
Pharmacogenomics holds the promise that drugs might one day be tailor-made for individuals and adapted to each person's own genetic makeup. Although factors like environment, diet, age, lifestyle, and state of health can influence a person's response to medicines, understanding an individual's genetic makeup is thought to be the key to creating personalized drugs with greater efficacy and safety. On these same principles one may also look forward to achieve anaesthesia customized for specific patient/ condition as per the patient's individualized genetic makeup, rather than adjusting the patient's physiology to routinely practiced anesthesia.

\section{Current applications of pharmacogenomics}

Today, clinical researchers use genetic tests for variations in cytochrome P450 genes to screen and monitor patients. In addition, many pharmaceutical companies screen their chemical compounds to see how well they are broken down by variant forms of CYP enzymes. ${ }^{15}$

\section{Conclusion}

The advances in field of genetics are opening new vistas and horizons of knowledge and foundations for us. As anaesthesiologist, are assuming role of peri-operative physicians, it has become imperative and essential for us to get deeper understanding in this new, exciting and challenging field,, so as to provide better perioperative care.

\section{Conflicts of interest}

Source of Funding: The authors declare that there was No source of any funding involved in the preparation of this manuscript. The authors confirm that there are no known conflicts of interest associated with this publication and there has been no significant financial support for this work that could have influenced its out-come.

\section{Authors' contribution}

The corresponding author conceptualized the main theme of the article. Second author did most of the literature search, correlating the findings from literature with the proposed hypothesis. The corresponding author did most of the writing, while second author did most of the editing, revising and final corrections in the preparation of manuscript.

\section{Declarations}

The authors want to confirm that both the authors had full access to all the data in the study and corresponding author takes the full and final responsibility for the decision to submit for publication.

\section{References}

1. Seck LJR, Sandford R. Molecular mechanisms of disease in Davidson's Principles and Practice of medicine. In: Boon NA, et al., editors. ( $2^{\text {nd }}$ edn), Churchill Livingstone: Toronto, Canada, 2009. p. 44.

2. Roden DM. Principle of clinical Pharmacology in Harrison's principles of Internal Medicinae. In: Kasper DL, et al., editors. $\left(16^{\text {th }}\right.$ edn), Macgraw-Hill New York, USA, 2005. p. 20.

3. Booij Leo H. Sex, age and geneticsin anesthesia. Curr Opin Anaesthesiol. 2008;21(4):462-466.

4. Butler MG, Hays BG, Hathaway MM, et al. Specific genetic diseases at risk for sedation/anesthesia complications. Anesth Analg. 2008;91(4):837-855.

5. Jones K. Smith's recognizable patterns of human malformation. (5th edn), WB Saunders, Philadelphia, USA, 1997. 
6. Wang CY, Chiu CL, Delilkan AE. Sevoflurane for difficult intubation in children. Br J Anaesth. 1998;80(3):408.

7. Magbagbeola JA. Abnormal responses to muscle relaxant in a patient with von Recklinghausen's disease (multiple neurofibromatosis). $\mathrm{Br} J$ Anaesth. 1970;42(8):710.

8. Manser J. Abnormal responses in von Recklinghausen's disease. $\mathrm{Br} J$ Anaesth. 1970;42:183.

9. Nagao H, Yamashita M, Shinozaki Y, et al. Hypersensitivity to pancuronium in a patient with von Recklinghausen's disease. $\mathrm{Br} J$ Anaesth. 1983;55(3):253.

10. Fink BR Shephard, Blandau RJ. Teratogenic Activity of Nitrous Oxide. Nature. 1967;214:146-148.
11. Smith G. Inhalational anaesthetic agents. In: Aitkenhead AR, et al., editors. Textbook of Anaesthesia. (3rd edn), Churchill Livingstone, New York, USA, 1996. p. 121-138.

12. Kallen B, Mazze RI. Neural Tube defects and first trimester operations. Teratology. 1990;41(9):717-720.

13. Sylvester GC, Khoury MJ, Lu X, et al. First trimester anesthesia exposure and the risk of central nervous system defects: a population based case- controlled study. Am J Pub Health. 1994;84(11):1757-1760.

14. Pharmacogenomics accessed on $26^{\text {th }}$ Dec 2015.

15. Hodgson J, Marshall A. Pharmacogenomics: will the regulators approve? Nature Biotechnolgy. 1998;16(3):243-246. 